\title{
Isolation of an actin promoter for strong expression of transgenes in the orchid genus Dendrobium
}

\author{
Ja Choon Koo
}

Received: 14 January 2013 / Accepted: 31 January 2013

(C) Korean Society for Plant Biotechnology

\begin{abstract}
We isolated and functionally characterized a Dendrobium Actin1 (DmACT1) promoter that drives strong gene expression in the orchid genus Dendrobium. A genomic fragment containing the region $3227 \mathrm{bp}$ upstream of the coding region of DmACT1 was obtained by inverse PCR. Detailed comparison of the full-length cDNA and genomic sequences revealed that $D m A C T 1$ has a 1374 bp first intron in the 5' UTR. However, the 5' flanking sequences upstream of the coding region showed no obvious sequence similarities compared to those of known promoters, including plant actin promoters. Serial deletion constructs of the 5' flanking region from the translation initiation codon were fused to the coding sequence of a GUS/luciferase fusion reporter to identify the regulatory elements necessary for promoter activity. Transient assays in the flowers of Dendrobium revealed that the 5' UTR-intron greatly enhanced promoter activity. Moreover, the DmACT1 promoter with its 5' UTR-intron yielded approximately 10-fold higher reporter activity than the rice Act1 promoter-intron. Our data suggest that the DmACT1 promoter with its 5' UTR-intron is a useful tool for strong expression of transgenes in Dendrobium orchids.
\end{abstract}

Keywords 5' UTR-intron, transient expression

Abbreviations CaMV, Cauliflower mosaic virus; GUS, $\beta$ -Glucuronidase; X-GlcA, 5-bromo-4-chloro-3-indolyl-ß-Dglucuronic acid cyclohexylammonium; DmACT1, Dendrobium moniliforme Actin1; RACE, rapid amplification of cDNA ends; UTR, untranslated region

J. C. Koo $(\bowtie)$

Division of Science Education and Institute of Science Education, Chonbuk National University, Jeonju, 561-756, Korea e-mail: jkoo@jbnu.ac.kr

\section{Introduction}

Orchids in the genus Dendrobium are some of the most important commercial orchid plants in the floricultural industry because many species in this genus produce a wide variety of beautifully colored flowers with unique morphologies and diverse fragrances. Furthermore, chemical compounds from orchids in this genus are used in traditional Chinese medicine (Wood 2006). Extensive conventional breeding programs based on intra- and inter-specific hybridization have been conducted over the last decade in Dendrobium species to improve floricultural traits such as color, plant shape, and growth. However, these approaches have resulted in limited success due to the lack of genetic resources, the sterility of inter-specific hybrids, and difficulty obtaining a genotype with all favorable genetic alleles present in the hybrids (Shibata 2008). Recently, technical advances in genetic transformation technology in Dendrobium have made it possible to modify target traits by direct incorporation of related genes with few undesirable effects on the existing plant phenotype (Chia et al. 1994; Kuehnle and Sugii 1992; Yang et al. 2003; Yu et al. 2001).

Strong, constitutive promoters are generally required for ectopic expression of target genes in a transgenic plant. Several promoters have been investigated as useful tools for strong expression of target genes in plants. Promoters obtained from CaMV $35 S$ RNA (Guilley et al. 1982), the maize polyubiquitin 1 ( Ubi 1) gene (Elliott et al. 1999), and the chimeric HBT promoter consisting of the CaMV $35 \mathrm{~S}$ enhancer fused to the basal promoter and the $5^{\prime}$ leader sequence of the maize C4PPDK gene (Sheen et al. 1995) have been evaluated in Dendrobium by genetic transformation or transient expression analysis (Chia et al. 1994; Tee et al. 2003; Yu et al. 2001). The HBT and Ubil promoters showed relatively low GUS expression compared to that of the CaMV $35 S$ promoter in Dendrobium sonia (Tee et al. 2003). Similarly, the rice Actinl (Act1) (McElroy 
et al. 1990) and maize Ubil promoters showed lower activity than the CaMV 35S promoter in some non-cereal monocot plants, namely Lolium, Liliaceae, and Gladiolus (Kamo et al. 1995; Wilmink et al. 1995). These results suggest that typical monocot promoters, such as Act1 and Ubil, although they perform well in some monocot species, do not perform well in all monocot species. In contrast, the CaMV $35 \mathrm{~S}$ promoter drove developmentaland tissue-specific expression of transgenes in transgenic rice and cotton plants, but was not constitutive (Sunilkumar et al. 2002; Tereda and Shimamoto 1990; Zhang and Wu 1988). Thus, for successful transformation of a target plant species, the promoter should be chosen with care.

Actin is a ubiquitous protein that plays essential roles in plant developmental processes such as cytoskeletal function, cell division, cell elongation, cellular trafficking, transportation of organelles, and pollen tube growth (Wasteneys and Galway 2003). High levels of Actin transcript were observed in all tissues and development stages in rice plants (McElroy et al. 1990). Likewise, the Actinl (Act1) promoter directed high GUS expression in all tissues of transgenic rice plants (Zhang et al. 1991). Recently, the rice Actin2 promoter, which drives high-level constitutive expression of GUS in transgenic rice, was also isolated for monocot transformations (He et al. 2009). However, the feasibility of using rice actin promoters in Dendrobium remains to be evaluated.

Some 5' UTR-introns, but not all, greatly enhance gene expression in humans (Jonsson et al. 1992), mice (Palmiter et al. 1991), Arabidopsis (Rose and Last 1997), rice (Jeon et al. 2001; Zhang et al. 1991), and Caenorhabditis elegans (Ho et al. 2001). Although 5' UTR-intron mediated gene expression (IME) exists in a diverse range of organisms, the precise mechanism underlying IME is likely not general. The length of 5' UTR-introns is highly variable across species, and 5' UTR-introns tend to be similar in size or larger than intragenic introns (Bradnam and Korf 2008). Moreover, it has been shown that the most highly expressed genes in humans and C. elegans tend to have shorter introns (Castillo-Davis et al. 2002), whereas in rice and Arabidopsis, highly expressed genes tend to have longer introns (Ren et al. 2006). Furthermore, analysis of the positional effect of IME has shown that IME effects decrease with distance from the transcription start site within a single gene in transgenic Arabidopsis (Rose 2004). Hence, the mechanisms underlying IME still remain to be elucidated.

One of the major barriers in genetic transformation of Dendrobium is the lack of promoters able to drive strong ectopic expression of target genes. To overcome this problem, we isolated the Actinl (DmACT1) promoter from a species of Dendrobium, because DmACT1 transcripts are abundantly expressed in the roots, leaves, and flowers of species in this genus. To identify the promoter region necessary for efficient gene expression, we examined the promoter activity of $D m A C T 1$ 5' flanking sequences using a GUS/luciferase fusion reporter system Transient assays in Dendrobium flowers revealed that the DmACT1 5' UTRintron is essential for transegene expression. Moreover, the $D m A C T 1$ promoter showed approximately 8- to 10 -fold higher active than the rice Actl promoter. Thus, the DmACTI promoter can be used to drive strong ectopic gene expression in Dendrobium orchids.

\section{Materials and methods}

\section{Plant Materials}

Dendrobium moniliforme "Jindo" (Whang et al. 2011) was cultivated under a 16 -h light $/ 8$-h dark cycle at $24^{\circ} \mathrm{C}$ in a greenhouse facility at Chonbuk National University. Leaf and root samples were harvested from young seedlings to isolate total RNA. Flower buds were harvested just after flowering in late May and throughout June for total RNA isolation or transient expression assays.

Northern blot analysis

Flowers, roots, and leaves were ground in liquid nitrogen using a mortar and pestle, and total RNA was isolated using Trizol reagent (Gibco BRL, NY, USA) according to the manufacturer's instructions. To prepare a DmACT1specificr probe, $120 \mathrm{bp}$ of the 5' UTR was amplified by radioactive PCR with the primers ActProbe-F (5'-GCCCT GCTAGTCGGCTGC-3') and ActProbe-R (5'-CTTTTACAA GCCTAAACAGAAG-3'). Five micrograms of total RNA was denatured and separated on a $2 \%$ agarose gel containing formaldehyde. After transferring the RNA onto a Hybond-N plus membrane (Amersham Biosciences, USA), the RNA was hybridized with ${ }^{32} \mathrm{P}$-labeled probe for $8 \mathrm{~h}$ at $65^{\circ} \mathrm{C}$ in Church buffer and washed as described previously (Koo et al. 2004). The blot was exposed to a Phosphor Screen (Molecular Dynamics, Amersham Bioscience, Inc.) to visualize the signal. The pixel intensity of each band was quantified and analyzed using TotalLab100 software (Nonlinear Dynamics, USA). The relative expression levels are shown as the ratio of the expression in the leaves to that in the other tissues. 
Cloning of partial Actin1 cDNA by degenerate RT-PCR

Partial DmACT1 cDNA was isolated using RT-PCR with degenerate primers. The primers dACT-F (5'-GARAARA TGACNCARATHATG-3') as the upstream forward primer and dACT-R (5'-TCNACRTCRCAYTTCATDAT-3') as the downstream reverse primer were designed based on a multiple alignment of orchid actin genes including those isolated from Dendrobium hybrid cultivars (EF612438.1 and GU301090.1), Phalaenopsis (AF246715.1), and Cymbidium (JQ360575.1 and JN177721.1). Five micrograms of total RNA was used to synthesize first strand cDNA using the SMARTer RACE cDNA Amplification Kit (Clontech Laboratories Inc., CA, USA) according to the manufacturer's instructions. PCR was performed with one-tenth of the synthesized cDNA, $20 \mu \mathrm{M}$ dACT-F, and $20 \mu \mathrm{M}$ dACT-R in $20 \mu \mathrm{l}$ of PCR premix (Clontech Laboratories Inc., CA, USA). The PCR conditions were as follows: initial denaturation at $95^{\circ} \mathrm{C}$ for $2 \mathrm{~min}, 30$ cycles of $94^{\circ} \mathrm{C}$ for $30 \mathrm{~s}$, $50^{\circ} \mathrm{C}$ for $30 \mathrm{~s}, 72^{\circ} \mathrm{C}$ for $1 \mathrm{~min}$, followed by a final extension of $72^{\circ} \mathrm{C}$ for $7 \mathrm{~min}$. PCR products were separated on a $0.8 \%$ agarose gel $(w / v)$. Specifically amplified DNA was recovered from the agarose gel using a PCR DNA purification Kit (GeneAll, Korea) and cloned into the pTOP TA V2 vector (Enzynomics, Inc., Korea). The nucleotide sequence of the insert was determined using vector- derived primers and analyzed using the BlastN program of NCBI (http://blast.ncbi.nlm.nih.gov/Blast.cgi).

Isolation of the full-length DmACT1 cDNA by 5'- and 3'-RACE

Full-length DmACT1cDNA was isolated by 5'- and 3'-RACE using the SMARTer RACE cDNA Amplification Kit according to the manufacturer's instructions with minor modifications. Briefly, $5 \mu \mathrm{g}$ of total RNA was used to synthesize first strand cDNA using the SMARTer RACE cDNA Amplification Kit (Clontech Laboratories Inc., CA, USA). One-tenth of the first strand cDNA was subjected to PCR with the DmACT1-specific reverse primer, ActRACE-R (5'-GTCGTGCGGCCACTAGCATATAG-3') for 5'-RACE or the forward primer, Act-RACE-F (5'-CTATT GGAGCTGGGAGATTTAGGTGC-3'), for 3'-RACE and vector derived universal primers to synthesize $D m A C T 1$, respectively. The PCR profile was as follows: initial denaturation at $95^{\circ} \mathrm{C}$ for 2 min followed by 30 cycles of $95^{\circ} \mathrm{C}$ for $15 \mathrm{~s}, 60^{\circ} \mathrm{C}$ for $30 \mathrm{~s}$, and $72^{\circ} \mathrm{C}$ for $1 \mathrm{~min}$. PCR products were purified using a PCR DNA purification Kit (GeneAll, Korea) and cloned into the pTOP TA V2 vector (Enzynomics, Inc., Korea) and sequenced with vector-derived primers. The complete DmACT1 cDNA sequence is available at Genbank (accession number KC171011, Fig. 1).

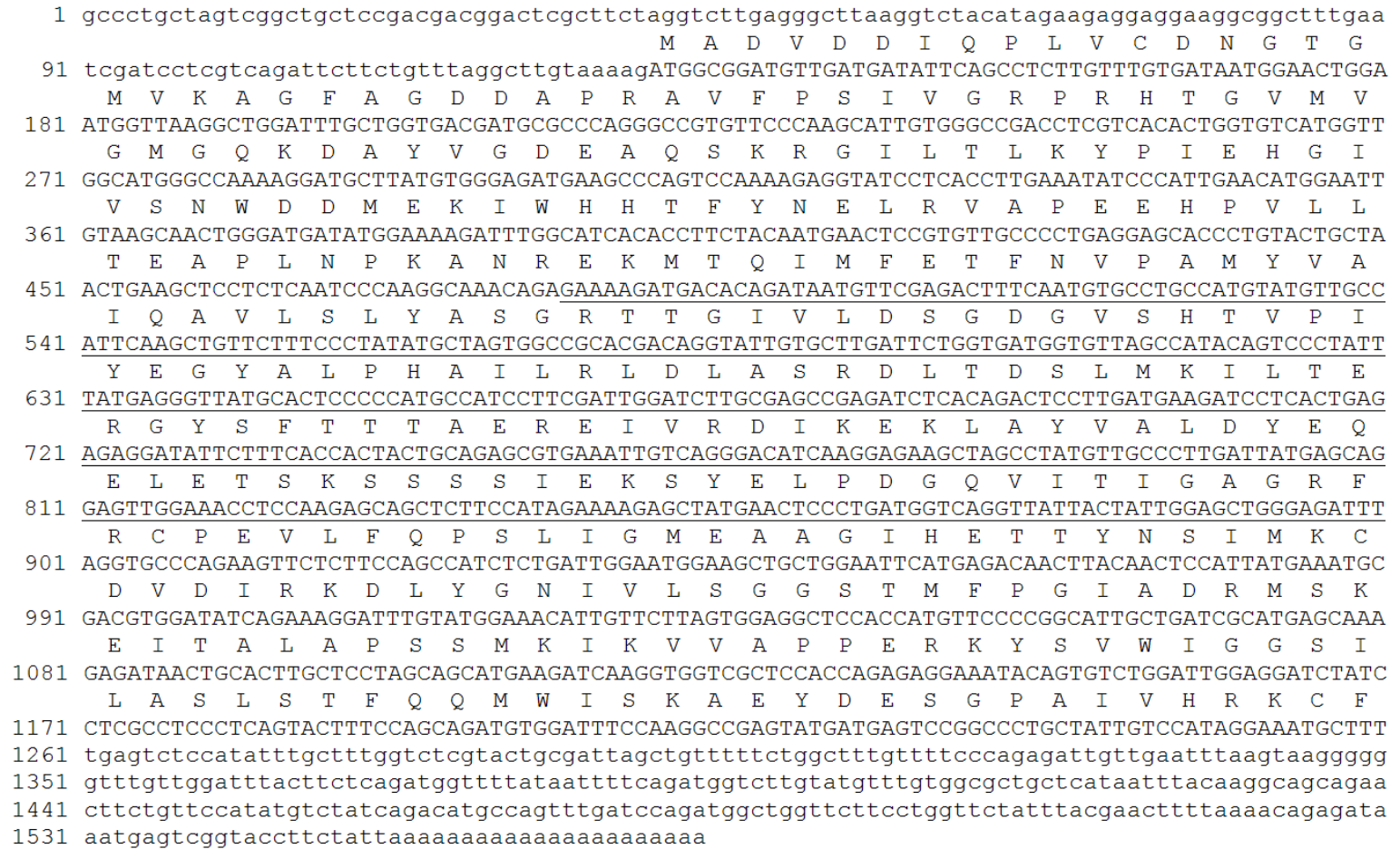

Fig. 1 Nucleotide and deduced amino acid sequence of DmACT1 (Genbank accession No. KC171011). The lower-case letters represent the $5^{\prime}$ and $3^{\prime}$ UTR sequences. Underlined sequences indicate the sequences isolated by degenerate RT-PCR 
Isolation of DmACT1 5'-flanking sequences by inverse PCR

Genomic DNA was extracted from fresh leaves using the CTAB method (Doyle and Doyle 1988). For inverse PCR, $1 \mu \mathrm{g}$ of genomic DNA was fully digested with Eco RI at $37^{\circ} \mathrm{C}$ for $4 \mathrm{~h}$ in a reaction volume of $100 \mu \mathrm{l}$. The digested DNA was extracted with a PCR DNA purification kit (GeneAll, Korea). The linearized DNA was then selfligated to form circular DNA with T4 DNA ligase (Enzynomics Inc., Korea) in a $10 \mu \mathrm{l}$ reaction volume at room temperature overnight. Self-ligated DNA was used as a template for inverse PCR. The PCR reaction was carried out in $40 \mu \mathrm{l}$ PCR mix containing $1 \mu \mathrm{l}$ of selfligated DNA and $20 \mu \mathrm{M}$ each of the inverse PCR primers, iACT-E-3-1 (5'-GCCATCCTTCGATTGGATCTTGCGAG CCG-3') and iACT-E-5-1 (5'-CTCTAAAACAAGCCAAA ACCCTCGCAG-3'). The PCR conditions were as follows: one cycle of initial denaturation at $95^{\circ} \mathrm{C}$ for $2 \mathrm{~min}$, followed by 30 cycles of $95^{\circ} \mathrm{C}$ for $15 \mathrm{~s}, 65^{\circ} \mathrm{C}$ for $30 \mathrm{~s}, 72^{\circ} \mathrm{C}$ for $3 \mathrm{~min}$, followed by $72^{\circ} \mathrm{C}$ for $5 \mathrm{~min}$. PCR products were purified using a PCR DNA purification kit and used as template for a second round of nested inverse PCR. The reaction mix and PCR condition were the same as those described above except the primers, iACT-E-3-2 (5'-CTCCT TGATGAAGATCCTCACTGAGAGAGG-3') and iACT-E-5-2 (5'- AAAGTAAGTCGCCTTATACCAAGGGCG -3') were used. Specifically amplified DNA was recovered from the agarose gel and cloned in the pTOP TA V2 vector for DNA sequencing. To obtain additional 5' flanking sequences of DmACT1, inverse PCR was conducted with Nde I-circularized genomic DNA as described above. The first round of PCR was performed using the primers iACT- a

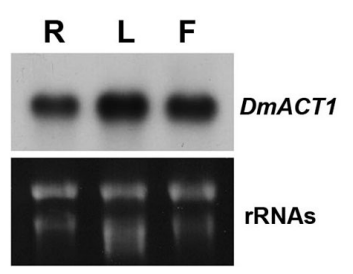

b

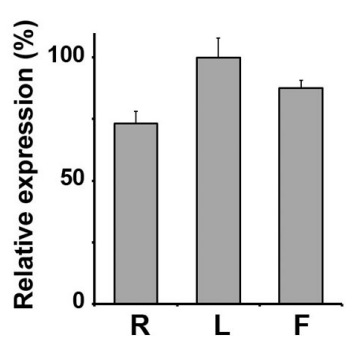

Fig. 2 Expression pattern of DmACT1 in Dendrobium. (a) RNA blot analysis of DmACT1. Total RNA was isolated from root (R), leaf (L), and flower (F) tissues. RNA was transferred to a membrane and hybridized with a ${ }^{32} \mathrm{P}$-labeled DmACT1 5' UTR probe. As a loading control, rRNAs was visualized after staining with ethidium bromide. (b) Quantification of DmACT1 expression. The DmACT1 band intensity from the RNA gel blot was measured and normalized to those of the 28S rRNA bands. Relative expression indicates the ratio of the value for the leaf to that of the other tissues. Bars indicate the SD of mean values
Nd-3-1 (5'-TCAAGGGTGGATCTGGAGCTACCA-3') and iACT-Nd-5-1 (5'-CAAAAGAACACAAACACCCAGATC TACC-3'). The second nested round of PCR was performed using the primers iACT-Nd-3-2 (5'- CCTGTTTGAATCG CGTTTGTAGCA-3') and iACT-Nd-5-2 (5'-CAGATCTA CCAAACGGCCCACCG-3'). Specifically amplified DNA was recovered from the agarose gel and cloned into the pTOP TA V2 vector for DNA sequencing. DNA sequencing was carried out using vector-derived primers and primers designed based on internal sequences, and both strands were sequenced. Nucleotide sequences were analyzed using the BlastN program of NCBI (http://blast.ncbi.nlm. nih.gov/Blast.cgi). A database search for promoter motifs and regulatory elements was performed using PlantCARE (http://bioinformatics.psb.ugent.be/webtools/plantcare/html).

\section{Vector construction}

The DmACT1 5' flanking sequence was deposited in Genbank (accession number KC171012, Fig. 3). A 3227 bp fragment of the DmACT1 5' flanking sequence, containing the promoter and the first intron sequence (from -1724 to +1503 relative to the transcription start site) was amplified by LA-PCR with the primers Act1-Nd (5'-ttggatcCATAT GCAAAGGACTAAACAAATTAAGTAG-3') and Act1int-R (5'-ttaagcttCTTTTACAAGCCTGAAGAATAACTG ACATG-3'). The underlined sequences indicate Bam HI and Hind III sites, respectively. The PCR product was digested with Bam $\mathrm{HI}$ and Hind $\mathrm{III}$ and cloned into the same sites in the GUS/Luciferase3300 vector, which encodes the GUS/Luciferase fusion reporter gene (Koo et al. 2007), to create $p D m A C T 1 N d$. In the same manner, the 1833 bp PCR product (from -1724 to +109 ) lacking the first intron sequence was amplified by PCR with the primers Act1-Nd and Act1-R (5'- ttaagcttAGAATCTGAC GAGGATCGATTCAAAGC-3'), and cloned in the GUS/ Luciferase 3300 vector to create $p D m A C T 1 N d \triangle i n t$. A 2555 bp fragment of the DmACT1 5' flanking sequence (from -1052 to +1503$)$ was amplified by PCR with the primers Act1-Xb (5'-ttggatcctTCTAGAAAAACTCGGTACAACT CTGTATTACC-3') and Actl-int-R, and cloned into the GUS/Luciferase3300 vector to create $p D m A C T 1 X b$. A 1602 bp fragment of the DmACT1 5' flanking sequence (from -99 to +1503$)$ was amplified by PCR with the primers, Act1-St (5'-ttggatccAGGCCTAAGCCCGTCTTGCTCAA $\left.-3^{\prime}\right)$ and Act1-int-R, and cloned into the GUS/Luciferase 3300 vector to create $p D m A C T 1 S t$. To compare with other monocot actin promoter, the Pst I and Hind III fragment of pCAMYS02 containing rice Actin1 promoter (a kind 
a

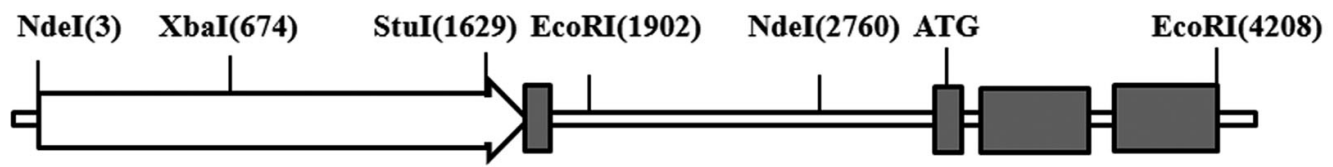

b

-1724 NdeI

$1 \stackrel{\text { CATATGCAAAGGACTAAACAAATTAAGTAGATTTTGGACTAATAATAATCACTTAATTTTCTAAAATGAGACATATTTGTATATATAATT }}{\longrightarrow}$

91 AсTTCATTTTCATTGATGAAAAGTCTTTTAAATAATAGTTAGTCACATCATTTAGATAAATtAAGAGATAAATAATATGATGGTTATCTT 181 GATTTAATGGGCCATATTTGTGAAAATGCTTTATTTTTAGTTTCATTGCACGACATATATGTGACACTCAATAATGGATCTGTAAAAATT 271 TGCTTATAGTTTTTAATTGCTAATTAAAATACATTTATTCATATTTTGTAGATAAATACATTATCTAATAATTAAATATTTGTTTCCTA 361 AAAGGGGACAATATGTTAATTGGTAAATTTTTCTTTATGCTCTCTAGTAGGAACTTTTATTTGGTCCGGTCACAGGACGTATCTCTAGGT 451 TCGGCCGCCAGTACAATTGCACCACGCGTTCTCACTAT TTACCGCGCGCCCCGACAACCCGACCCGGAACGGACCCATGATGGCTAGATA

541 TTTCTCGATTTTGCCAGGTATTGCTCGATATTACTCGGTATTGCTAGGTATTGGTCGATTTTGCTTGAGAATAACCAATACCGACCAAAT -1051 XbaI

631 ACCGACCAAATACCGAGCAAATACTGAGTTGTACCGAGTTTT

721 CAGAGCGGTATTTCTCGTTATTGCCATGCAATACCTAGCAATACCTAGCAAACACCGAGTAATACATCGCAATGCGCGAACATAGCATTC 811 TCGGGTTTCGGTATGGGTCAAGTCGTCGGGCCGCACGATAGAGAGCAAGAGCATGTGGCGACATGTTAT TGGCGGACGAACGTAGCTCTA 901 CGTCCGGTCAGAGGACGTAATAATTGTTCCTCTAGTAGAGTTGAGCAAAAAATTATGTGTTGCGTGCTAAAAAAATT TGAGTCCATAGAG 991 TTTATGCTTTACATCTTAGGATGTACAAACATTCAGCACGAACAGTTGCAATTTAGTGGCTCATTAGTGTATTAATTAGGGATCTGATTA 1081 TTACATAACGTTGAAAATTTGATTAATTCATTGATTAGGAAAGCATTGATT GACTTTTCACTTTTATTTGCGTTTCAAATTT GAAAATAA 1171 GGGCCTAAGTGGTGTTGGATGTAAGAGAGCTAGAAAGAGCCAATTGTAACTTATTTTTCTTCACATTATAGTGAAATTAGCGTCTCCCCA 1261 TGCACGTAGACATATATGCCGAACCACGTAAATCGTCATGCTCTTTATGTTGATTGTGCTTTGATTCTTATTTTGTTTATGCTATTTGGA 1351 CAACATAATTAATATTTTTTGTACAACGCTTGCTTCCCTTTTGATTCT TTCACCTTAAGCAAACTATAGATGTGGCAAAAGTTTTACAAT 1441 TTTTTTTCTGCAAAAAATAAATATATGATACCCTACGAGGCAAAGTTAACTAACTAGATTAACAAGTAATAAAAAATTGTTTAAGTAAA 1531 TAAAAGCCCAATGGCCAACATGCCAGCTCGTGGAGCCCTTGTGGTCCTTCAAGATTGGACCGCGATCTTAACGAGGAGGAGAGCCCGAAA -99 StuI TATA 1621 TCCTAAGGCCTAAGCCCGTCTTGCTCAATTATTTCCACAACTGATTCCTCTTCTCACTT GACCAACCGCTCGATCTATAAATTGCCCAGT $+1$ 1711 TTCСTCAСАTTTATGCCCTGCTAGTCGGCT GCTCCGACGACGGACTCGCTTCTAGGTCTTGAGGGCTTAAGGTCTACATAGAAGAGGAGG $+109$

1801 AAGGCGGCTTTGAATCGATCCTCGTCAGATTCTTCTGTTTAG/gtaagggtttctct tatctagatctgtgattgttgtt tatatctagat

EcoRI

1891 ttatttcactgaattcggtgggccgtttggtagatctgggtgtttgtgttcttttgaatgtgttttttgaatttcatagatatttggat 1981 gatcgggaagtatggacttgtttttagcggatgagcttgatttccaatgtagatctatgactttgcttttaggaaagagatgcatgat 2071 ttgatttcgccettggtataaggcgacttacttatttgaaagatcagatttactgcgagggttttggcttgttttagagatttattat 2161 tttcaagggtggatctggagctaccactatatctcctgtttgaatcgcgtttgtagcatgatatcttgcgacttccctgggaaaaactta 2251 aagcgtttatctaacctatatttgttggacgtttagttgcactagtttgacgtcttcatttccttcattttttttcttttgagattt 2341 ggtagaggctataaatactgtgaccatttatcggcagatttgttgattttacacagattgttagcatcatattcatcaggaaatgactg 2431 ataaatgcttataaacttctatgaggaggtcccatgtttatcgccagatttgcttattttacacagattattaacatttaattcgtcag 2521 gaaatgactgattaaagtgctttacttctatgaggaggtcccatgcacgatataaaatactaaaggattctccttggattctgtggttat 2611 tttctggaagccagttgttgttgttgtatggattggtctgatctgttgtgaaccaatgaatctttatcaaattttgttggatagtttcat NdeI

2701 gcaggtgcctattagttctcctgcatcaagtcatgcttagactttaccgttgaaaacatatgtcaacatggtttcagataccaaactca 2791 tcattgaaatgtattcttcttatagtgtgaagtaaagtttccttttagtaacttgctaatttgtatgttgttggagatgaaatccctttc 2881 aggttgctgatgttcatacaatccaatttctggtatggattttagcttgtgtaggttgtaaattgtgccttgaaatgtttaggttacttt 2971 ctcaagcaacttagattccaaattacaagtgtagaacagtgtagataagtagatataagtatctttggacactttgttgcatgccattt 3061 actttcgctaatattggactatgttggtttgttgcttgaattgcaattgcgagataactgctttgttcatgaagcagatctgatgagt

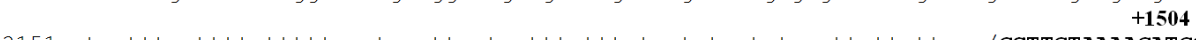

3151 atgattggtttcttttaaatggattcatggttcttctcatatcatgtcagttattcttcag/GCTTGTAAAAGATGGCGATGTTG

3241 ATGATATTCAGCCTCTTGTTTGTGATAATGGAACTGGAATGGTTAAGGTAagctccaacctaagttgtagtctggaaatagcaccaattg 3331 cgtccgactt tcct $c t t t t t t$ t t t $g t t a g$ GCTGGATTTGCTGGTGACGATGCGCCCAGGGCCGTGTTCCCAAGCATTGTGGGCCGA 3421 CCTCGTCACACTGGTGTCATGGTTGGCATGGGCCAAAAGGATGCTTATGTGGGAGATGAAGCCCAGTCCAAAAGAGGTATCCTCACCTTG 3511 AAATATCCCATTGAACATGGAATTGTAAGCAACTGGGATGATATGGAAAAGATTTGGCATCACACCTTCTACAATGAACTCCGTGTTGCC 3601 CCTGAGGAGCACCCTGTACTGCTAACTGAAGCTCCTCTCAATCCCAAGGCAAACAGAGAAAAGATGACACAGATAATGTTCGAGACTTTC

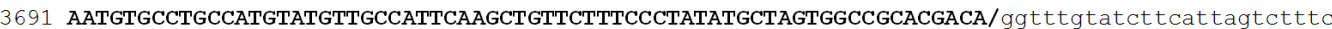

3781 tgctat cacattt tcatct tcagatggtctcCcactgaaact ttgcttgcttgta/GGTATTGTGCTTGATTCTGGTGATGGTGTTAGCC 3871 ATACAGTCCCTATTTATGAGGGTTATGCACTCCCCCATGCCATCCTTCGATTGGATCTTGCGAGCCGAGATCTCACAGACTCCTTGATGA 3961 AGATCCTCACTGAGAGAGGATATTCTTTCACCACTACTGCAGAGCGTGAAATTGTCAGGGACATCAAGGAGAAGCTAGCCTATGTTGCCC 4051 TTGATTATGAGCAGGAGTTGGAAACCTCCAAGAGCAGCTCTTCCATAGAAAAGAGCTATGAACTCCCTGATGGTCAGGTTATTACTATTG 4141 GAGCTGGGAGATTTAGGTGCCCAGAAGTTCTCTTCCAGCCATCTCTGATTGGAATGGAAGCTGCTGGAATC EcoRI

Fig. 3 Physical map and nucleotide sequence of the 5' flanking region of DmACT1. (a) Physical map of the 5' flanking region of $D m A C T 1$. Open arrow and gray boxes indicate the promoter region and exons of DmACT1, respectively. ATG indicates the translation initiation site. (b) Nucleotide sequence of the 5' flanking region of DmACT1 (Genbank accession No. KC171012). Primers used for subsequent construction of promoter truncation vectors are indicated by arrows. The restriction sites used in inverse PCR are underlined. The transcription initiation site is designated as +1 and is underlined. The translation initiation site is indicated by an underlined M. The TATA box is underlined. Bold upper-case and lower-case letters represent exon and intron sequences, respectively. Slashed lines indicate exon-intron boundaries. Note that the first intron resides in the 5' UTR

gift from In-Sun Yoon, RDA, Korea) with its first intron was cloned in the same sites of GUS/Luciferase 3300 vector to create $p$ Actl. The DNA sequences of all constructs were confirmed by DNA sequencing analysis. These constructs were used for transient luciferase reporter assays in Dendrobium flower cells. 
Microprojectile bombardment

Promoter activity of constructs containing various lengths of DmACT1 5' flanking sequence were examined by a microprojectile bombardment-mediated transient assay (Whang et al. 2011). Approximately $3.5 \mu \mathrm{g}$ of gold particles (0.6 $\mu \mathrm{m}$, Bio-Rad Laboratory, USA) were coated with $5 \mu \mathrm{g}$ of DNA construct. As an internal control, $1 \mu \mathrm{g}$ of CaMV35S promoter-Renilla luciferase construct (a kind gift from $\mathrm{H}$. G. Nam, DGIST, Korea) was co-bombarded. Flowers were bombarded with the DNA construct-coated gold particles at a helium gas pressure of 1,000 Psi and a target distance of $6 \mathrm{~cm}$.

\section{Analysis of Gus/luciferase activity}

The reporter activity of the GUS/luciferase fusion protein was examined as described previously (Koo et al. 2007). Briefly, the bombarded samples were transferred to a 24well plate containing $0.5 \mathrm{mM}$ D-luciferin or coelenterazine in MS medium, and bioluminescence was measured using a high-performance CCD camera (VersArray system, Roper Scientific Ltd., UK). Images were analyzed by MetaVue version 5.2 software (Universal Imaging Corporation, USA). To normalize the actin promoter activity, the ratio of firefly luciferase activity was calculated and represented by comparison with that of the control Renilla luciferase. Data are shown as mean values obtained from three independent microprojectile bombardment experiments. Histochemical staining of GUS activity was performed by overnight incubation in X-GlcA (Duchefa, The Netherlands) substrate solution as described previously (Koo et al. 2007). When necessary, samples were bleached with $70 \%$ ethanol.

\section{Results}

Molecular cloning of full-length DmATC1 cDNA

To isolate an actin gene from Dendrobium, we used a degenerate PCR approach using primers designed to DNA sequences conserved among orchid actin genes (See Materials and Methods). We cloned a 513 bp partial cDNA fragment, which we named $D m A C T 1$ (Fig. 1). Then, we isolated and characterized the 1573 bp full-length DmACT1 cDNA by 5' and 3' RACE PCR. RACE PCR was conducted using the SMARTer RACE cDNA amplification kit that allows the cloning of the full-length sequence of a target transcript. Sequence analysis indicated that $D m A C T 1$ contains a 129 bp 5' UTR, a 1131 bp coding sequence, and a 288 bp 3' UTR (Fig. 1). A database search revealed that the nucleotide and deduced amino acid sequences of DmACT1 were the most similar to the nucleotide and amino acid sequences of Phalaenopsis ACT2 (Accession No. AF246715.1) ( $87 \%$ and $99 \%$, respectively).

\section{Expression of DmACT1 in Dendrobium}

To determine whether DmACT1 expression was ubiquitous in Dendrobium, we performed RNA gel blot analysis using total RNA isolated from roots, leaves, and flowers. Actins are ubiquitous proteins in plant cells and are encoded by a multigene family (Šlajcherová et al. 2012). Because coding sequences are highly conserved among actin genes, the 5' UTR of DmACT1 was amplified for use as a probe in RNA gel blot analysis. As shown in Fig. 2, DmACT1 transcripts were abundantly expressed in all organs that we examined. Although transcript levels were slightly higher in leaf than the other tissues, our results nevertheless indicate that $D m A C T l$ is ubiquitously expressed in Dendrobium.

Isolation of genomic fragments containing the 5' flanking region of DmACT1

The 5' flanking sequences of DmACTl were isolated by two round of inverse PCR. Circularized genomic DNA digested with Eco RI was used as a template in the first round of inverse PCR. However, a sequence comparison with the full-length DmACT1 cDNA revealed that the isolated genomic clone contained only the exon-intron boundaries and the truncated form of the intron without the sequence corresponding to the 5' UTR (Fig. 3). Thus, we isolated upstream sequences by a second round of inverse PCR using circularized genomic DNA digested with Nde I. Figure 3 shows the full-length sequence of the isolated genomic clone. Sequence analysis revealed that the genomic clone contained a 3216 bp region upstream of the coding sequence of DmACT1. Because the nucleotide sequence of the full-length DmACT1 cDNA matched the base at position 1725 exactly, this was designated +1 as the putative transcription start site. In a motif search, a TATA box sequence was found between bases -29 and -23. The translation initiation codon was found at position +1504 in the second exon. Interestingly, the 5' UTR of $D m A C T 1$ contains a 1374 bp long intron from +119 to +1492 . However, the 5' UTR-intron of DmACT1 is somewhat longer than that reported in other plant species (Bradnam and Korf 2008), and shared no sequence similarity with known sequences in a database search. 
DmACT1 promoter analysis by GUS/luciferase reporter transient assays

5' UTR-introns can enhance promoter activity in animals and plants (Bradnam and Korf 2008). Thus, we generated four promoter constructs with or without the 5' UTR-intron to examine the promoter activity of the 5 ' flanking sequence of DmACT1.

For analysis of promoter activity, choice of reporter system is very important to avoid artifacts arising from the stability or multimerization of reporter protein. We previously demonstrated that the GUS/luciferase fusion protein has the advantages of both GUS and luciferase proteins, namely high resolution histochemical GUS staining and real-time bioluminescence imaging with a luciferin-dependent rapid turn-over rate (Koo et al. 2007). Hence, we used the GUS/luciferase fusion reporter system for analysis of 5' flanking sequence of DmACT1. As shown in Figure 4, a $2778 \mathrm{bp}$ fragment of the flanking sequence of DmACT1 5' (from -1724 to +1054 ) was fused to the coding sequence of the GUS/luciferase reporter gene to create $p D m A C T 1 N d$. To examine the effect of the 5' UTR-intron on gene expression, we fused the $1833 \mathrm{bp}$ fragment $(-1724$ to +105$)$ lacking the 5' UTR-intron to the coding sequence of the reporter gene to create $p D m A C T 1 N d \triangle i$ int. Similarly, $p D m A C T 1 X b$ and $p D m A C T 1 S t$ constructs containing 2105 bp (-1051 to +1054) and 1153 bp (-99 to +1054) fragments of the 5' flanking sequence of $D m A C T 1$, respectively, were generated by fusion with the coding sequence of Gus/ luciferase.
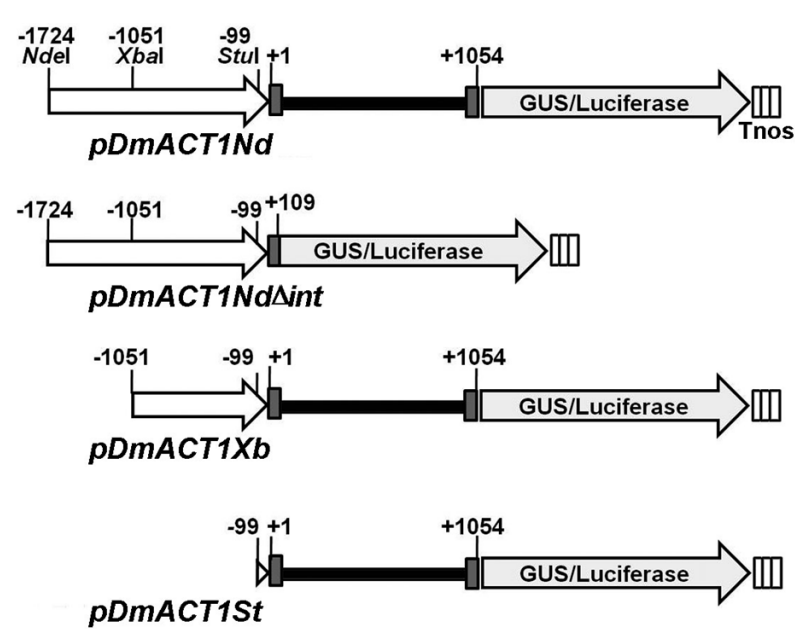

Fig. 4 Physical map of the DmACT1 5' flanking sequence-GUS/ luciferase fusion constructs. Various portions of the 5'-flanking and/or 5'-noncoding UTRs of the DmACT1 gene were fused to the coding sequence of a GUS/luciferase fusion reporter gene. White arrows show the promoter region. Gray and black boxes represent the exon and 5'UTR intron, respectively
The flower is a very important plant characteristic in the floricultural industry and genetic manipulation of orchids. Therefore, many researches primarily target the improvement of flower traits. RNA blot analysis indicated that $D m A C T 1$ is also highly expressed in flowers (Fig. 2). Thus, we analyzed the promoter activity of the 5' flanking sequence of DmACT1 in flowers of Dendrobium by transient expression assays.

Since transient expression system was highly affected by DNA concentration, efficiency of bombardment and conditions of plant samples, CaMV35S promoter-Renilla luciferase construct was used in the experiment as an internal control to normalize the promoter activity. An unbombarded sample was used as a negative control. The rice actin promoter ( $p$ Act1), which is widely used in monocot plants, was also included in the experiment to compare promoter activities. The promoter activity of each bombarded sample was monitored by the time-lapse bioluminescence imaging using a high-performance CCD camera. Then, the value of firefly luciferase activity driven by actin promoter constructs was normalized with that of Renilla luciferase driven by CaMV35S promoter. As shown in Figure 5, flowers bombarded with pDmACT1Nd and $p D m A C T 1 X b$ containing the 5' UTR-intron showed high levels of luciferase activity, although $p D m A C T 1 N d$ had 1.5 -fold higher activity than $p D m A C T 1 X b$. Luciferase activity driven by $p D m A C T 1 N d$ and $p D m A C T 1 X b$ increased gradually after bombardment, and steady-state levels were reached after 28 to $30 \mathrm{~h}$ of incubation. Furthermore, pDmACT1Nd showed 8- to 10-fold higher luciferase activity than the rice actin promoter ( $p A c t 1)$. However, flowers bombarded with $p D m A C T 1 N d \triangle i n t$ displayed undetectable levels of luciferase activity. These results suggest that the 5' UTR-intron is necessary for efficient promoter activity of DmACT1. Flowers bombarded with pDmACT1St, which lacks the large portion of promoter region, showed near basal-levels of luciferase activity.

The promoter activity of the 5 ' flanking sequence of DmACT1 was further confirmed by histochemical GUS staining. Similar with luciferase activity, the strong histochemical GUS stains were observed in flowers bombarded with $p D m A C T 1 N d$ and $p D m A C T 1 X b$, whereas no detectable activity was observed in flowers bombarded with $p D m A C T 1 N d \triangle i n t$ and $p D m A C T 1 S t$. These results confirm that the 5' UTR-intron is necessary for efficient promoter activity of DmACT1. As expected, the rice Act1 promoter (pAct1) displayed weaker GUS stains than the DmACT1 5, flanking sequence. Taken together, our results demonstrate that the 5'-flanking sequence of DmACT1 can promote strong gene expression in Dendrobium. 

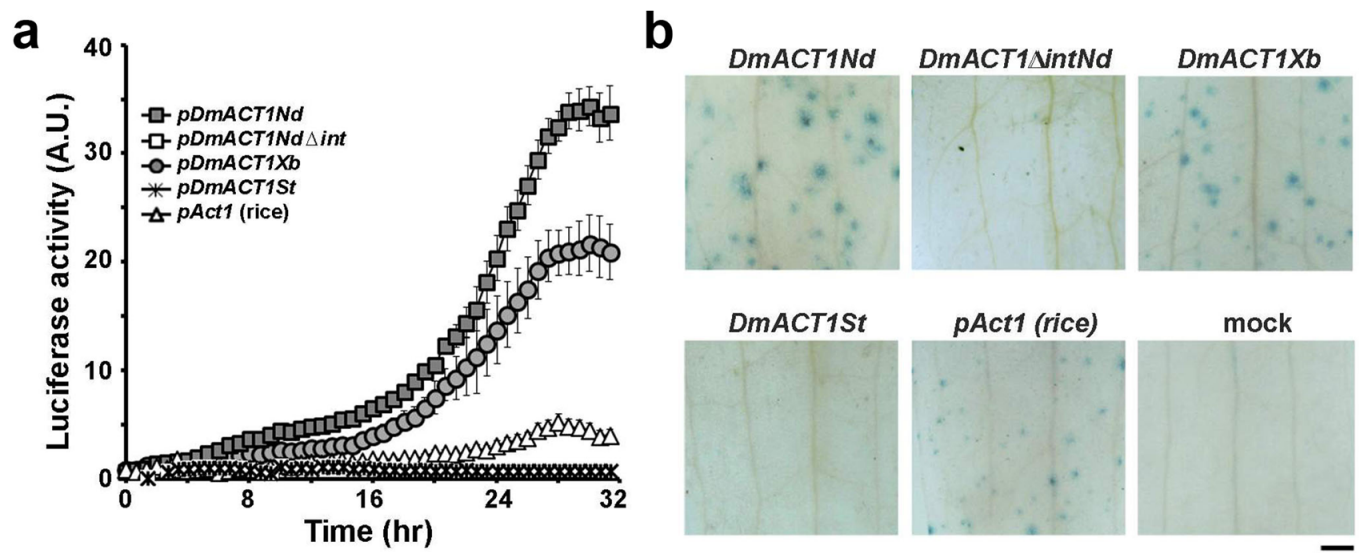

Fig. 5 Analysis of transient GUS/luciferase activity driven by various portions of the DmACT1 5'-flanking sequence in Dendrobium flowers. Each construct was co-bombarded with pCaMV35S-Renilla luciferase. Expression of the promoter from the rice actin promoter ( $p$ Act 1$)$ in Dendrobium was also evaluated for comparison purposes. (a) Relative luciferase activity in bombarded flowers. Bombarded sample was divided into two parts and transferred to medium containing luciferin and coelenterazine in a 24 -well plate. Bioluminescence was measured using a high-performance cool CCD every $40 \mathrm{~min}$ for $32 \mathrm{hr}$. The values were subtracted from those of unbombarded samples (mock) and normalized relative to the Renilla luciferase activity. Note that $p D m A C T 1 N d \triangle I n t$ and $p D m A C T 1 S t$ showed background levels of bioluminescence. Bars indicate SD of the mean value $(n=3)$. (b) GUS histochemical staining obtained after $32 \mathrm{hr}$ of incubation. Representative images from three independent experiments are shown. The scale bar indicates $1 \mathrm{~mm}$

\section{Discussion}

Promoters that drive strong and constitutive gene expression are important for plant biotechnology applications. Several promoters have been developed for use in monocot transformation including maize Ubil (Elliott et al. 1999) and rice Act1 (McElroy et al. 1990). Although these promoters drive strong expression of genes in rice, they show lower activity than the CaMV $35 \mathrm{~S}$ promoter in some monocot plants (Kamo et al. 1995; Wilmink et al. 1995). This is also the case in Dendrobium. GUS expression driven by the Ubil promoter was lower than that of GUS expression driven by the CaMV $35 S$ promoter in Dendrobium sonia (Tee et al. 2003). Furthermore, the efficiency of the Act1 promoter in orchids, including Dendrobium, has not been evaluated. Actin genes belong to a multigene family in plants, with some actin isoforms constitutively and strongly expressed in plants (An et al. 1996; McElroy et al. 1990; Šlajcherová et al. 2012). In this study, we demonstrated that $D m A C T 1$ expression is ubiquitous and abundant in the flowers, leaves, and roots of Dendrobium by RNA blot analysis (Fig. 1). Thus, we isolated the DmACT1 promoter to determine if it could drive strong expression of a reporter gene in Dendrobium.

We isolated the 5'-flanking sequence of DmACT1 by two round of inverse PCR. Sequence analysis indicated that it contained a $1374 \mathrm{bp}$ intron in the 5' UTR region. To examine if this region had promoter activity, we fused various lengths of DmACT1 5' flanking sequence to a GUS/ luciferase fusion reporter (Koo et al. 2007) to monitor promoter activity by real-time luciferase imaging and to assess GUS activity by histochemical staining. We also utilized a transient expression system for promoter analysis. Although cellular expression patterns can be determined in stable transgenic plants in contrast to transiently transfected plants, undesirable effects may occur in measurement of promoter activity due to the presence of flanking sequences in the T-DNA insertion site, multiple gene copies, and silencing or co-suppression of genes. Transient GUS/ luciferase expression analysis revealed that the DmACT1 5 ' flanking sequence had 8- to 10 -fold higher promoter activity in the flower organs of Dendrobium orchids than the rice actin promoter ( $p A c t 1)$. Hence, the $D m A C T 1$ promoter can be used to drive strong ectopic expression of transgenes in Dendrobium.

It has been shown that some 5' UTR-introns contribute to high levels of transgene expression in both dicot and monocot transgenic plans including Arabidopsis, maize, and rice (Bradnam and Korf 2008; Jeon et al. 2001; Lu et al. 2008; Zhang et al. 1991; Rose and Last 1997). Although the underlying mechanism remains unclear, it has been shown that the effect of the 5' UTR-intron decreases gradually as its distance from the translation initiation site increases (Rose 2004). Furthermore, the 5' UTR-intron does not drive gene expression when located in the 3' UTR. Second, the IME effect of the 5' UTR-intron is not general to all organisms. The rice Actl intron and the maize Ubil intron greatly enhances gene expression in rice, but have negligible effects in non-cereal monocot plants (Kamo et al. 1995; Tee et al. 2003; Wilmink et al. 
1995). Moreover, the combination of the CaMV35S promoter and maize Adh15' UTR-intron resulted in decreased GUS expression in the protoplasts of sugarcane (Wilmink et al. 1995), whereas addition of the maize shrunken-1 locus 5' UTR-intron to the same promoter resulted in greatly increased CAT gene expression in the protoplasts of various grass species (Vasil et al. 1989). Third, IME differs between plant and animal cells. Highly expressed genes have a shorter 5' UTR-intron in animal cells than plant cells, and vice versa (Castillo-Davis et al. 2002; Ren et al. 2006). Fourth, the effect of IME may be due to transcriptional or post-transcriptional events. In some cases, IME has been shown to be related to increased enzyme activity or protein accumulation rather than increased mRNA levels (Bourdon et al. 2001; Kamo et al. 2012; Mascarenhas et al. 1990; Samadder et al. 2008; Rose 2004). Moreover, the effect of the 5' UTR-intron of rice ubiquitin (rub3) on promoter activity has been shown to be tissue- and cell-type specific ( $\mathrm{Lu}$ et al. 2008). Thus, it would be interesting to explore the IME mechanism of the 5' UTR-intron in DmACT1 in future studies. Taken together, our data suggest that the $D m A C T 1$ promoter will be of great use in orchid biotechnology as it drives high levels of transgene expression, and represents a good genetic resource for the development of even more efficient orchid promoters.

\section{Acknowledgements}

The author wish to thank Drs. Hong Gil Nam, Ju-Kon Kim and In Sun Yoon for generously providing Renilla luciferase construct and rice actin promoter. The author would like to thank Dr. Moon-Sik Yang, Hyo-Yeon Moon and Ki-Nam Kim for kindly providing equipment and technical assistance in the experiment of microprojectile bombardment. This work was supported by grants from Korea National Arboretum (the development and conservation of useful plant resources of in and outside the country, 2012).

\section{References}

An YQ, McDowell JM, Huang S, McKinney EC, Chambliss S, Meagher RB (1996) Strong, constitutive expression of the Arabidopsis ACT2/ACT8 actin subclass in vegetative tissues. Plant J 10:107-121

Bourdon V, Harvey A, Lonsdale DM (2001) Introns and their positions affect the translational activity of mRNA in plant cells. EMBO Rep 2: 394-398
Bradnam KR, Korf I (2008) Longer first introns are a general property of eukaryotic gene structure. PloS one 3: e3093.

Castillo-Davis CI, Mekhedov SL, Hartl DL, Koonin EV, Kondrashov FA (2002) Selection for short introns in highly expressed genes. Nat Genet 31:415-418

Chia TF, Chan YS, Chua NH (1994) The firefly luciferase gene as a non-invasive reporter for Dendrobium transformation. Plant J 6:441-446

Doyle JJ, Doyle L (1990) Isolation of plant DNA from fresh tissue. Focus 12:13-15

Elliott AR, Campbell JA, Dugdale B, Brettell, RIS, Grof CPL (1999) Green fluorescent protein facilitates rapid in vivo detection of genetically transformed plant cells. Plant Cell Rep 18:707-714

Guilley H, Dudley RK, Jonard G, Balfizs E, Richards KE (1982) Transcription of cauliflower mosaic virus DNA: Detection of promoter sequences, and characterization of transcripts. Cell 30:763-773

He C, Lin Z, McElroy D, Wu R (2009) Identification of a rice actin2 gene regulatory region for high-level expression of transgenes in monocots. Plant Biotechnol J 7:227-239

Ho SH, So GM, Chow KL (2001) Postembryonic expression of Caenorhabditis elegans mab-21 and its requirement in sensory ray differentiation. Dev Dyn 221: 422-430

Jeon JS, Lee S, Jung KH, Jun SH, Kim C, An G (2000) Tissuepreferential expression of a rice alpha-tubulin gene, OsTubA1, mediated by the first intron. Plant Physiol 123: 1005-1014

Jonsson JJ, Foresman MD, Wilson N, McIvor RS (1992) Intron requirement for expression of the human purine nucleoside phosphorylase gene. Nucleic Acids Res 20: 3191-3198

Kamo K, Blowers A, Smith F, Van Eck J, Lawson R (1995) Stable transformation of Gladiolus using suspension cells and callus. J Am Soc Hort Sci 120:347-352

Kamo K, Kim AY, Park SH, Joung YH (2012) The 5' UTR-intron of the Gladiolus polyubiquitin promoter GUBQ1 enhances translation efficiency in Gladiolus and Arabidopsis. BMC Plant Biol 12:79

Koo J, Kim Y, Kim J, Yeom M, Lee IC, Nam HG (2007) A GUS/luciferase fusion reporter for plant gene trapping and for assay of promoter activity with luciferin-dependent control of the reporter protein stability. Plant Cell Physiol 48:1121-1131

Koo JC, Asurmendi S, Bick J, Woodford-Thomas T, Beachy RN (2004) Ecdysone agonist-inducible expression of a coat protein gene from tobacco mosaic virus confers viral resistance in transgenic Arabidopsis. Plant J 37:439-448

Kuehnle AR, Sugii N (1992) Transformation of Dendrobium orchid using particle bombardment of protocorms. Plant Cell Rep 11:484-488

Lu J, Sivamani E, Li X, Qu R (2008) Activity of the 5' regulatory regions of the rice polyubiquitin rubi3 gene in transgenic rice plants as analyzed by both GUS and GFP reporter genes. Plant Cell Rep 27:1587-1600

Mascarenhas D, Mettler IJ, Pierce DA, Lowe HW (1990) Intronmediated enhancement of heterologous gene expression in maize. Plant Mol Biol 15: 913-920 
McElroy D, Rothenberg M, Reece KS, Wu R (1990) Characterization of the rice actin gene family. Plant Mol Biol 15: 257-268

Palmiter RD, Sandgren EP, Avarbock MR, Allen DD, Brinster RL (1991) Heterologous introns can enhance expression of transgenes in mice. Proc Natl Acad Sci U S A 88: 478-482

Ren X-Y, Vorst O, Fiers MWEJ, Stiekema WJ, Nap J-P (2006) In plants, highly expressed genes are the least compact. Trends Genet 22:528-532

Rose AB (2004) The effect of intron location on intron-mediated enhancement of gene expression in Arabidopsis. Plant $\mathrm{J}$ 40:744-751

Rose AB, Last RL (1997) Introns act post-transcriptionally to increase expression of the Arabidopsis thaliana tryptophan pathway gene PAT1. Plant J 11: 455-464

Samadder P, Sivamani E, Lu J, Li X, Qu R (2008) Transcriptional and post-transcriptional enhancement of gene expression by the 5' UTR intron of rice rubi3 gene in transgenic rice cells. Mol Genet Genomics 279:429-439

Sheen J, Hwang S, Niwa Y, Kobayashi H, Galbraith DW (1995) Green fluorescent protein as a new vital marker in plant cells. Plant J 8:777-784

Shibata, M. (2008) Importance of genetic transformation in ornamental plant breeding. Plant Biotech 25:3-8

Šlajcherová K, Fišerová J,Fischer L,Schwarzerová K (2012) Multiple actin isotypes in plants: diverse genes for diverse roles? Front Plant Sci. 3:226

Sunilkumar G., Mohr LA, Lopata-Finch E, Emani C, Rathore KS (2002) Developmental and tissue-specific expression of CaMV 35S promoter in cotton as revealed by GFP. Plant Mol Biol 50: 463-474

Tee CS, Marziah M, Tan CS, Abdullah MP (2003) Evaluation of different promoters driving the GFP reporter gene and selected target tissues for particle bombardment of Dendrobium Sonia 17. Plant Cell Rep 21: 452-458

Tereda R, Shimamoto K (1990) Expression of CaMV 35S-GUS gene in transgenic rice plants. Mol Gen Genet 220:389-392

Vasil V, Clancy M, Ferl RJ, Vasil IK, Hannah LC (1989) Increased gene expression by the first intron of maize shrunken-1 locus in grass species. Plant Physiol 91:1571-1579

Wasteneys GO, Galway ME (2003) Remodeling the cytoskeleton for growth and form: an overview with some new views. Ann Rev Plant Biol 54:691-722

Wilmink A, Van de Ven BCE, Dons JJM (1995) Activity of constitutive promoters in various species of the Liliaceae. Plant Mol Biol 28:949-955

Whang SS, Um WS, Song IJ, Lim PO, Choi K, Park KW, Kang KW, Choi MS, Koo JC (2011) Molecular analysis of anthocyanin biosynthetic genes and control of flower coloration by Flavonoid 3', $5^{\prime}$-Hydroxylase $\left(\mathrm{F}^{\prime} 5^{\prime} \mathrm{H}\right)$ in Dendrobium moniliforme. J Plant Biol 54: 209-218

Wood HP (2006) The Dendrobiums. AR. G. Gantner Verlag, Ruggell, Leichenstein.

Yang SH, Yu H, Goh CJ (2003) Functional characterisation of a cytokinin oxidase gene DSCKX1 in Dendrobium orchid. Plant Mol Biol 51:237-248

Yu H, Yang SH, Goh CJ (2001) Agrobacterium-mediated transformation of a Dendrobium orchid with the class 1 knox gene DOH1. Plant Cell Rep 20:301-305

Zhang W, Wu R (1988) Efficient regeneration of transgenic plants from rice protoplasts and correctly regulated expression of the foreign gene in the plants. Theor Appl Genet 76: 835-840

Zhang W, McElroy D, Wu R (1991) Analysis of rice Act 15 ' region activity in transgenic rice plants. Plant Cell 3:1155-1165 